\title{
Crosstalk between the interleukin-6 (IL-6)-JAK-STAT and the glucocorticoid-nuclear receptor pathway: synergistic activation of IL-6 response element by IL-6 and glucocorticoid
}

\author{
T Takeda, H Kurachi, T Yamamoto, Y Nishio, Y Nakatsuji, \\ K-i Morishige, A Miyake and Y Murata
}

Department of Obstetrics and Gynecology, Osaka University Medical School, Osaka 565-0871, Japan

(Requests for offprints should be addressed to T Takeda, Department of Obstetrics and Gynecology, Osaka University Medical School, 2-2 Yamadaoka, Suita, Osaka 565-0871, Japan)

\begin{abstract}
Cytokines and steroid hormones use different sets of signal transduction pathways, which seem to be unrelated. Interleukin-6 (IL-6) uses JAK tyrosine kinase and STAT (signal transducer and activator of transcription) transcription factor. Glucocorticoid binds glucocorticoid receptor (GR), which is a member of the steroid receptor superfamily. We have studied the crosstalk between the IL-6-JAK-STAT and glucocorticoid-nuclear receptor pathways. IL-6 and glucocorticoid synergistically activated the IL-6 response element on the rat $\alpha_{2}$-macroglobulin promoter (APRE)-driven luciferase gene. The exogenous expression of GR enhanced the synergism. The exogenous expression of dominant negative STAT3 completely abolished the IL-6 plus glucocorticoid-induced activation of the APRE-luciferase gene. Tyrosine phosphorylation of STAT3 stimulated by IL-6 alone was not different from that by IL-6 plus glucocorticoid. The protein level of
\end{abstract}

STAT3 was also not increased by glucocorticoid stimulation. The time course of STAT3 tyrosine phosphorylation by IL-6 plus glucocorticoid was not different from that by IL-6 alone. The synergism was studied on the two other IL-6 response elements, the junB promoter (JRE-IL-6) and the interferon regulatory factor-1 (IRF-1) promoter (IRF-GAS) which could be activated by STAT3. The synergistic activation by glucocorticoid on the IL-6activated JRE-IL-6 and the IRF-GAS-driven luciferase gene was not detected. Glucocorticoid did not change the mobility of IL-6-induced APRE-binding proteins in a gel shift assay. These results suggest that the synergism was through the GR and STAT3, and the coactivation pathway which was specific for APRE was the target of glucocorticoid.

Journal of Endocrinology (1998) 159, 323-330

\section{Introduction}

Interleukin-6 (IL-6) is a cytokine that plays an important role in host defense mechanisms such as the synthesis of acute phase proteins (Gauldie et al. 1989). However, maximal expression of several acute phase protein genes (such as $\alpha_{2}$-macroglobulin, $\alpha_{1}$-acid glycoprotein and fibrinogen) requires stimulation by glucocorticoid in addition to that by IL-6 (Baumann et al. 1987, Baumann 1989). Glucocorticoid binds glucocorticoid receptor (GR), a member of the nuclear receptor superfamily and regulates the transcription of glucocorticoid responsive genes (Evans 1988). The GR is widely distributed in nearly all cell types and has an essential role in cell metabolism and growth.

In the case of the $\alpha_{1}$-acid glycoprotein gene, an example of the synergistic action of IL-6 and glucocorticoid, IL-6 acts through the IL-6 response element and glucocorticoid through the glucocorticoid response element (GRE)
(Baumann et al. 1990, Nishio et al. 1993). The combination of two response elements generates a synergistic interaction. However, in the $\alpha_{2}$-macroglobulin gene, it is reported that only the acute phase response element (APRE) site is sufficient to confer a synergistic induction by IL-6 and glucocorticoid (Hocke et al. 1992). The detailed mechanism of this synergistic activation remains unclear.

Cytokines and steroid hormones use different sets of signal transduction pathways, which seem to be unrelated. IL-6 uses JAK tyrosine kinases (JAK1, JAK2, Tyk2) and STAT (signal transducer and activator of transcription) transcription factor (STAT3) signal transduction pathway, and activates the IL-6 response elements such as the APRE in the rat $\alpha_{2}$-macroglobulin promoter (Wegenka et al. 1993, Lutticken et al. 1994, Zhong et al. 1994, Nakajima et al. 1995). Glucocorticoid binds GR and activates the transcription through GRE (Beato et al. 1995). 
In this study, we examined the crosstalk between the IL-6-JAK-STAT pathway and glucocorticoid-nuclear receptor pathway. We further analyzed the mechanism of this regulation.

\section{Materials and Methods}

\section{Cells and cell culture}

The human breast carcinoma cell line MCF7 was cultured in DMEM medium (Gibco BRL, Life Technologies, Inc., Grand Island, NY, USA) containing 10\% fetal calf serum (CSL, Parkville, Victoria, Australia) (Takeda et al. 1997b).

\section{Plasmids}

The $4 \times$ APRE-luciferase gene containing four repeats of APRE (IL-6 response element of the rat $\alpha_{2}$-macroglobulin promoter), was constructed by subcloning two repeats of the oligonucleotides (5'-TCGACATCCTTCTGGG AATTCTGATCCTTCTGGGAATTCTGGGTAC -3') (Hocke et al. 1992) in front of the minimal junB promoterluciferase gene as described (Takeda et al. 1994). The $4 \times$ mAPRE-luciferase gene containing four repeats of mutated APRE (mAPRE) was constructed by subcloning two repeats of the oligonucleotides $\left(5^{\prime}\right.$-TCGACATCCT TCTCTAGATTCTGATCCTTCTCTAGATTCTGG GTAC- $3^{\prime}$ ) in front of the minimal junB promoterluciferase gene (Takeda et al. 1994). The $3 \times \mathrm{JRE}-\mathrm{IL}-6-$ luciferase gene containing three repeats of JRE-IL-6 (IL-6 response element of the junB promoter) was constructed by subcloning three repeats of the oligonucleotides (5'-GCGCTTCCTGACAGTGACGCGAGCCG-3') (Nakajima et al. 1993) in front of the minimal junB promoter-luciferase gene as described (Takeda et al. 1994). The $3 \times$ IRF-GAS-luciferase gene containing three repeats of IRF-GAS (IL-6 response element of the interferon regulatory factor-1 (IRF-1) promoter) was constructed by subcloning three repeats of the oligonucleotides (5'-TGATTTCCCCGAAATGACGGCACGC-3') (Kojima et al. 1996) in front of the minimal junB promoter-luciferase gene. The human glucocorticoid receptor (hGR) expression vector was pRShGR $\alpha$ (Hollenberg et al. 1987). The dominant negative STAT3 expression vector was pCAGGS-HA-STAT3D (Nakajima et al. 1996).

\section{DNA transfection and luciferase assays}

DNA transfection and luciferase assays were performed as described (Nakajima et al. 1993, Takeda et al. 1994). In each experiment, cells were seeded at $3 \times 10^{5}$ per $6 \mathrm{~cm}$ dish and $24 \mathrm{~h}$ later $2.9 \mu \mathrm{g}$ luciferase reporter plasmid, 1.0 $\mu \mathrm{g}$ pEFLacZ (Nakajima et al. 1993) (internal control for transfection efficiency), and $2 \cdot 1 \mu \mathrm{g}$ expression vector were transfected as required. The total amount of the transfected DNA was adjusted to $6 \cdot 1 \mu \mathrm{g}$ with pTZ19R (Pharmacia, Milwaukee, WI, USA). Cells were extracted, and luciferase activities were assayed in a MicroLumat luminometer (EG \& G Berthold, Postfach, Germany) as described (Takeda et al. 1994). $\beta$-Galactosidase activity was determined to normalize the transfection efficiency. All experiments were performed in triplicate and repeated at least three times with essentially similar results. Results are expressed as relative luciferase activity. Data are shown as averages of three independent experiments, with standard deviations indicated by bars.

\section{Cytokines and antibodies}

Cytokines used in this study were human recombinant IL-6 (a gift from Dr T Hirano, Osaka University Medical School, Osaka, Japan). The antibodies used for Western blotting and immunoprecipitation were antiphosphotyrosine monoclonal antibody (4G10, Upstate Biotechnology Inc., Lake Placid, NY, USA) and antiSTAT3 polyclonal antibody (C-20, Santa Cruz Biotechnology, Inc., Santa Cruz, CA, USA).

\section{Immunoprecipitation, SDS-PAGE and Western blotting}

Cells $\left(2 \times 10^{7}\right)$ were harvested and lysed for $60 \mathrm{~min}$ in $1 \mathrm{ml}$ lysis buffer (50 mM Tris (pH 7.5), $150 \mathrm{mM} \mathrm{NaCl}$, $1 \mathrm{mM}$ EDTA, $0 \cdot 5 \% \mathrm{NP}-40,1 \mathrm{mM}$ sodium orthovanadate, $1 \mathrm{mM} \mathrm{NaF}, 0 \cdot 75 \mathrm{mM}$ phenylmethylsulfonyl fluoride, $15 \%$ glycerol, and $10 \mu \mathrm{g} / \mathrm{ml}$ each of aprotinin, pepstatin and leupeptin) as described (Lutticken et al. 1994). Proteins in the lysates from $2 \times 10^{7}$ cells were immunoprecipitated with the anti-STAT3 polyclonal antibody. Immune complexes were separated by SDS-PAGE (7.5\%) and transferred to an Immobilon-P nylon membrane (Nihon Millipore Ltd, Tokyo, Japan) which was immunoblotted with the relevant primary antibody as described (Takeda et al. 1994). Proteins were detected by enhanced chemiluminescence (ECL, Amersham International plc, Amersham, Bucks, UK). Rehybridization was performed as described (Ruff Jamison et al. 1993).

\section{Electrophoretic mobility shift assays (EMSAs)}

Nuclear and cytoplasmic extracts were prepared from MCF7 cells according to the method of Sadowski et al. (1993). Double-stranded oligonucleotides used as probes in the EMSAs were as follows: 5'-GCGCCTTCTGGGA ATTCCTA-3', 5'-GCGCTAGGAATTCCCAGAAG3' (Takeda et al. 1994). Binding reactions and electrophoresis proceeded as described (Nakajima et al. 1993). In the supershift assays, anti-STAT3 polyclonal antibody (C-20, Santa Cruz Biotechnology, Inc.) or anti-GR 
(A)

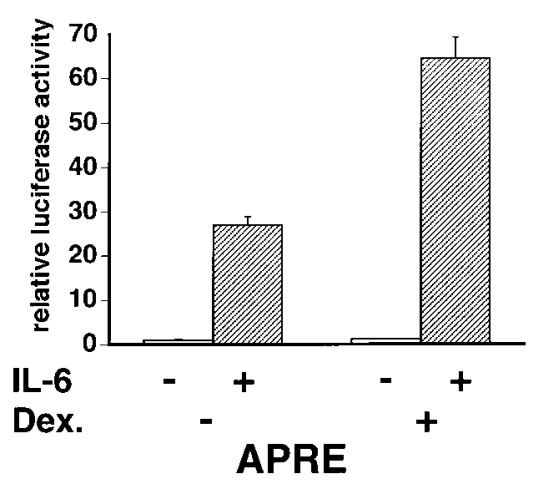

(B)

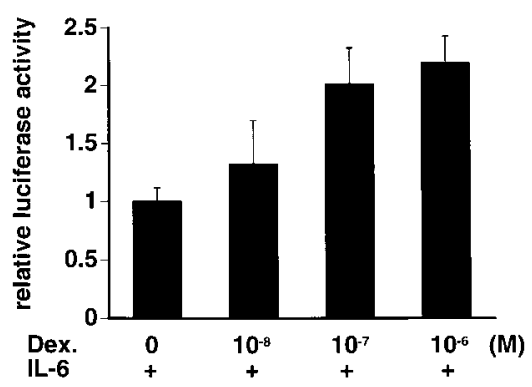

(C)

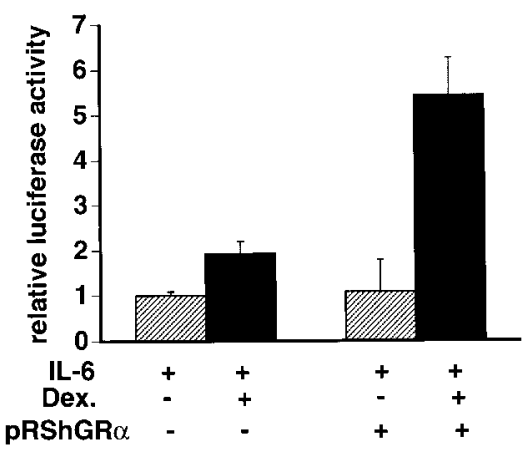

Figure 1 The synergistic effect by IL-6 and Dex on the APRE-luciferase gene activation. (A) MCF7 cells were transfected with the $4 \times$ APRE-luciferase gene. The transfected cells were incubated with vehicle $(-)$ or IL-6 $(100 \mathrm{ng} / \mathrm{ml})$ alone or in combination with Dex $\left(10^{-6} \mathrm{M}\right)$ for $6 \mathrm{~h}$, and luciferase activity was determined. (B) Dose-dependent effect of Dex on the IL-6-induced APRE activation. MCF7 cells were transfected with $4 \times$ APRE-luciferase gene, then stimulated with IL-6 $(100 \mathrm{ng} / \mathrm{ml})$ and Dex (at the concentrations indicated) for $6 \mathrm{~h}$, and luciferase activities were determined. (C) Exogenous expression of GR enhanced the synergistic action of Dex on the IL-6-activated APRE-luciferase gene. MCF7 cells were transfected with $4 \times$ APRE-luciferase gene and the GR expression vector $(\mathrm{pRShGR \alpha})$. The transfected cells were stimulated with vehicle $(-)$ or IL-6 $(100 \mathrm{ng} / \mathrm{ml})$ alone or in combination with Dex $\left(10^{-6} \mathrm{M}\right)$ for $6 \mathrm{~h}$, and luciferase activity was determined. Data are expressed in relative luciferase activity. Mean value in the vehicle treated group was arbitrarily determined as 1 in (A). That in the IL-6 treated group without Dex stimulation was arbitrarily shown as 1 in (B) and that without exogenous GR expression as 1 in (C). Data are averages of three independent experiments, with standard deviations indicated by bars.

polyclonal antibody (Affinity Bioreagents, Inc., Golden, CO, USA) was added to the binding reaction mixture.

\section{Results}

\section{IL-6 and glucocorticoid synergistically activate the} APRE-luciferase gene

We tested the IL-6-induced activation of the APREluciferase gene with or without dexamethasone (Dex). The $4 \times$ APRE-luciferase plasmid was transiently transfected into MCF7 cells, and luciferase activity was assayed after $6 \mathrm{~h}$ of IL-6 stimulation (Fig. 1A). Transfection efficiency was normalized by $\beta$-galactosidase activity as described in Materials and Methods. The basal APRE activity was not significantly activated by Dex stimulation $(1 \cdot 1-$ fold $)$. The IL-6-induced APRE activity was synergistically enhanced by Dex stimulation (from 26.8-fold by IL-6 alone to $64 \cdot 4$-fold by IL-6 plus Dex). The $4 \times$ mAPRE-luciferase plasmid was also transiently transfected, and luciferase activity was assayed after $6 \mathrm{~h}$ of IL-6 stimulation with or without Dex. As IL-6 or Dex stimulation and IL-6 plus Dex stimulation did not activate the mAPRE-luciferase gene at all (data not shown), the synergism is dependent on the APRE site.

We examined the dose dependence of the synergistic action by Dex (Fig. 1B). Dex synergistically enhanced the IL-6-activated APRE gene transcription in a dosedependent manner. A significant synergistic action of Dex on the IL-6-activated APRE was detected from $10^{-7} \mathrm{M}$ Dex stimulation. Next we examined whether or not the exogenous expression of GR enhanced the synergistic action (Fig. 1C). The synergistic enhancement by Dex on the IL-6-activated APRE-luciferase gene expression was 2.0-fold without exogenous GR, whereas it increased to 5.4-fold with exogenous GR expression. These results suggested that the synergistic effect of glucocorticoid is through the GR.

\section{The synergistic action of glucocorticoid requires the STAT3}

It has been reported that a mutant of STAT3 (STAT3D, in which two critical residues in the DNA-binding domain, E434 and E435, have been changed to aspartates) efficiently and specifically inhibits IL-6-induced APREluciferase gene activation and acts as a dominant negative STAT3 (DN-STAT3) (Nakajima et al. 1996). We examined the effect of the exogenous expression of STAT3D on the synergistic action of Dex (Fig. 2). STAT3D efficiently inhibited the APRE activation by both IL- 6 alone and IL-6 plus Dex. These facts suggested that the effect of glucocorticoid is through the activated STAT3.

\section{The synergistic action of glucocorticoid is very rapid and long}

It is well known that the IL-6-JAK-STAT signal transduction pathway is very rapid and does not require protein synthesis (Nakajima et al. 1993, 1995, Hirano et al. 1994). We examined the time course of the synergistic action of Dex on the IL-6-activated APRE activation (Fig. 3). The synergistic action of Dex was detected from $3 \mathrm{~h}$ of Dex stimulation. Though the IL-6-induced APRE activation 


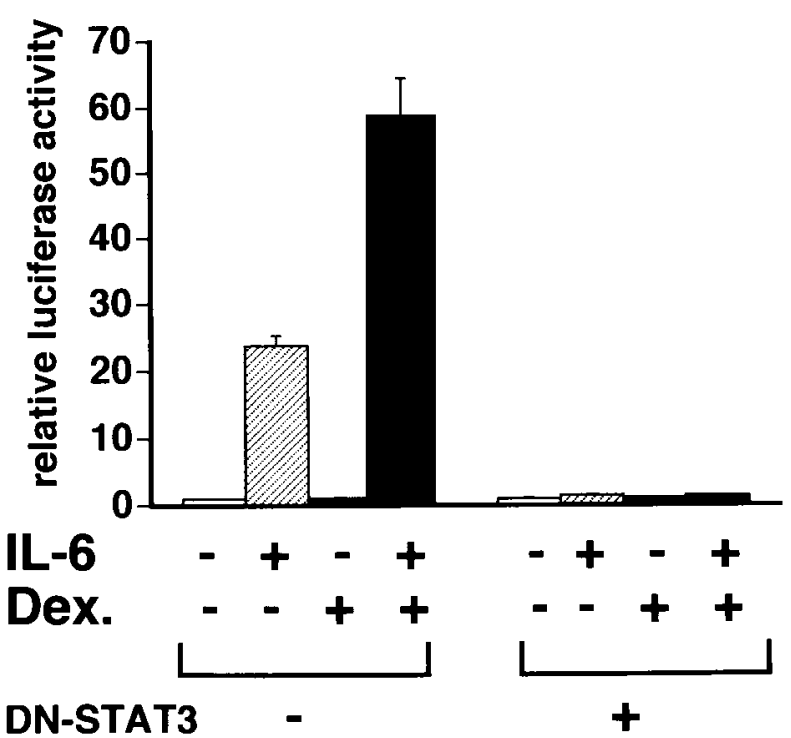

Figure 2 DN-STAT3 repressed the synergistic activation of IL-6-activated APRE by Dex. MCF7 cells were transfected with $4 \times$ APRE-luciferase gene and the DN-STAT3 expression vector, pCAGGS-HA-STAT3D. The transfected cells were stimulated with vehicle $(-)$ or IL-6 $(100 \mathrm{ng} / \mathrm{ml})$ alone or in combination with Dex $\left(10^{-6} \mathrm{M}\right)$ for $6 \mathrm{~h}$, and luciferase activity was determined. Relative luciferase activity in the unstimulated cells is arbitrarily shown as 1 . Data are averages of three independent experiments, with standard deviations indicated by bars.

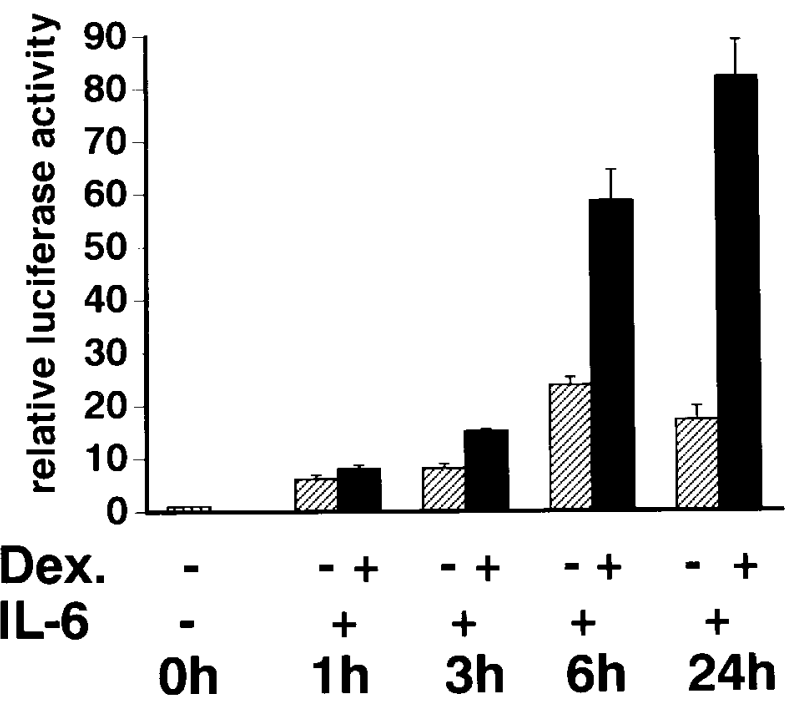

Figure 3 Time course of the synergistic enhancement by Dex on the IL-6-activated APRE gene transcription. MCF7 cells were transfected with the $4 \times$ APRE-luciferase gene. The transfected cells were incubated with vehicle $(-)$ or IL-6 $(100 \mathrm{ng} / \mathrm{ml})$ alone or in combination with Dex $\left(10^{-6} \mathrm{M}\right)$ for $0,1,3,6$ and $24 \mathrm{~h}$ and luciferase activity was determined. Relative luciferase activity in the unstimulated cells is shown as 1 . Data are averages of three independent experiments, with standard deviations indicated by bars.
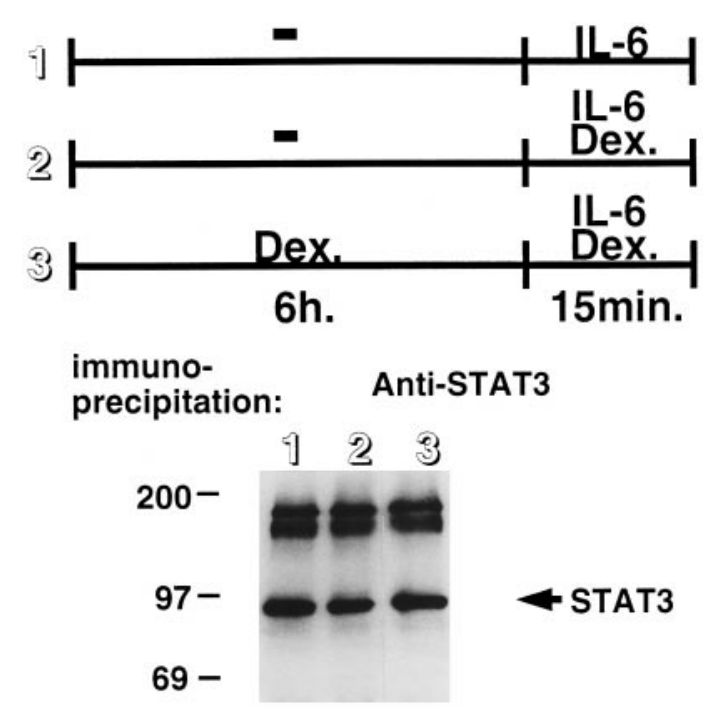

immuno- Anti-
blot:

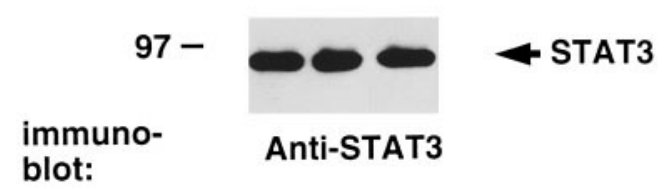

Figure 4 Absence of the enhancing effect of Dex on the IL-6-induced tyrosine phosphorylation of STAT3. MCF7 cells were treated for $15 \mathrm{~min}$ with IL-6 (100 ng/ml) alone (lane 1), IL-6 $(100 \mathrm{ng} / \mathrm{ml})$ plus Dex $\left(10^{-6} \mathrm{M}\right)$ (lane 2$)$ or pretreated for $6 \mathrm{~h}$ with $\operatorname{Dex}\left(10^{-6} \mathrm{M}\right)$ and then treated for $15 \mathrm{~min}$ with IL-6 $(100 \mathrm{ng} / \mathrm{ml})$ plus Dex $\left(10^{-6} \mathrm{M}\right)$ (lane 3). Cell lysates were immunoprecipitated with anti-STAT3 antibody. Immune complexes were separated by SDS-PAGE and blotted with anti-phosphotyrosine antibody (upper panel). The blots were then stripped and reprobed with anti-Stat3 antibody (lower panel).

reached maximal levels within $6 \mathrm{~h}$ stimulation, the synergistic effect of Dex continued increasing at $24 \mathrm{~h}$. These results indicated that the effect of Dex on the IL-6-JAK-STAT pathway was very rapid and lasted for a long time.

Glucocorticoid stimulation does not change the tyrosine phosphorylation and the protein level of STAT3

We analyzed which part of the IL-6-JAK-STAT pathway was activated by Dex stimulation. The IL-6-induced tyrosine phosphorylation of STAT3 is very rapid and transient (Nakajima et al. 1995), and the level of phosphorylation reached its maximum at $15 \mathrm{~min}$ in MCF7 cells (see later Fig. 5A). We examined whether IL-6 plus Dex stimulation $(15 \mathrm{~min})$ enhanced the tyrosine phosphorylation of STAT3, compared with that by IL-6 alone (15 min) (Fig. 4 lanes 1 and 2). IL-6 with or without Dex 
A immunoprecipitation:

Anti-STAT3

IL-6

$0153060120 \mathrm{~min}$.

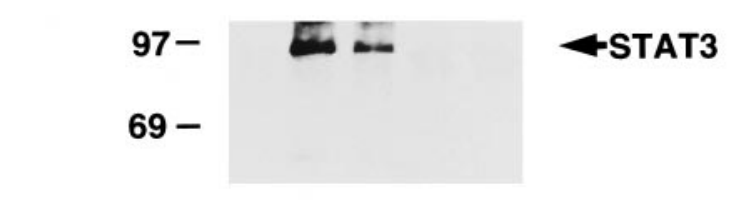

immunoblot:
$B$ immunoprecipitation:
Anti-STAT3

IL-6 +Dex.

$0153060120 \mathrm{~min}$.

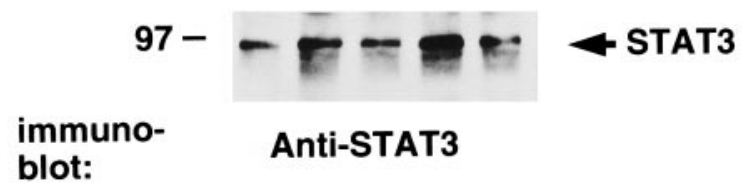

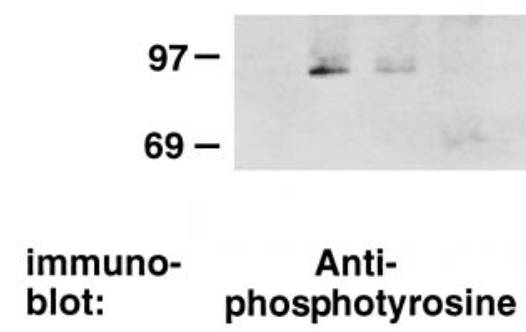

\&STAT3

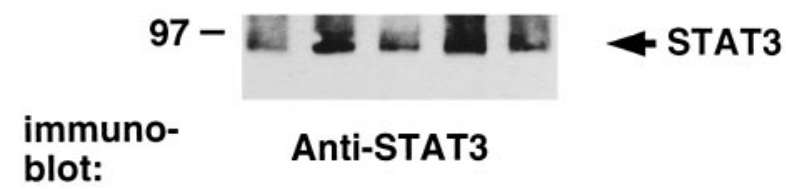

Figure 5 Dex did not change the time course of the IL-6-induced STAT3 tyrosine phosphorylation. MCF7 cells were treated for the indicated times with (A) IL-6 $(100 \mathrm{ng} / \mathrm{ml})$ or $(B) \mathrm{IL}-6(100 \mathrm{ng} / \mathrm{ml})$ plus Dex $\left(10^{-6} \mathrm{M}\right)$. Cell lysates were immunoprecipitated with anti-STAT3 antibody. Immune complexes were separated by SDS-PAGE and blotted with anti-phosphotyrosine antibody (upper panels of $\mathrm{A}$ and $\mathrm{B})$. The blots were then stripped and reprobed with anti-Stat3 antibody (lower panels of A and B).

induced phosphorylation of STAT3 to a similar extent (Fig. 4, upper panel, lanes 1 and 2). Dex also did not alter the protein level of STAT3 (Fig. 4, lower panel, lanes 1 and 2). Next we examined whether the pretreatment of Dex $(6 \mathrm{~h})$ enhanced the tyrosine phosphorylation or increased the protein level of STAT3 (Fig. 4, lane 3). The phosphorylation and the protein level of STAT3 were not changed by Dex pretreatment. These results indicated that Dex did not enhance the IL-6-induced STAT3 phosphorylation.

It is possible that the Dex treatment may change the time course of STAT3 phosphorylation, Dex may sustain the IL-6-induced STAT3 phosphorylation and enhance the IL-6-induced APRE activation. Therefore we examined whether the Dex treatment sustains the IL-6-induced tyrosine phosphorylation of STAT3 (Fig. 5A and B). Tyrosine phosphorylation of STAT3 with IL-6 plus Dex treatment was very rapid and transient; the level of phosphorylation reached maximum at 15 min stimulation, and it returned to basal levels at $60 \mathrm{~min}$ and did not change thereafter until $120 \mathrm{~min}$. We could not detect the IL-6induced tyrosine phosphorylation of STAT3 with or without Dex even after $24 \mathrm{~h}$ (data not shown). The time course was not different from that by IL-6 alone. These results indicated that the Dex treatment did not change the time course of IL-6-induced STAT3 phosphorylation.
Glucocorticoid does not enhance the IL-6-induced activation of the JRE-IL-6 and IRF-GAS-luciferase genes

The above data suggest that Dex enhances the IL-6induced APRE activation by stimulating a pathway other than the tyrosine phosphorylation of STAT3. There are several IL-6 response elements which could be activated by tyrosine phosphorylated STAT3 (Kojima et al. 1996). The regulation mechanism of these response elements other than the tyrosine phosphorylation of STAT3 remains unclear (Nakajima et al. 1995, Zhang et al. 1995). Two other IL-6 response elements, JRE-IL-6 and IRF-GAS, are activated in a manner different from that of APRE (Kojima et al. 1996). Therefore, we examined the effect of Dex treatment on the IL-6-induced activation of JRE-IL6-luciferase and IRF-GAS-luciferase genes (Fig. 6). The $3 \times$ JRE-IL-6-luciferase and $3 \times$ IRF-GAS-luciferase plasmids were transiently transfected into MCF7 cells, and luciferase activity was assayed after $6 \mathrm{~h}$ of IL- 6 alone or IL-6 and Dex stimulation. IL-6 efficiently activated both response elements, but Dex treatment did not enhance the IL-6-induced activation of JRE-IL-6 and IRF-GASluciferase genes as effectively as that in the APREluciferase gene. These results indicated that the synergistic effect of Dex on the IL-6-JAK-STAT pathway is specific to APRE activation. The coactivation pathway which is 


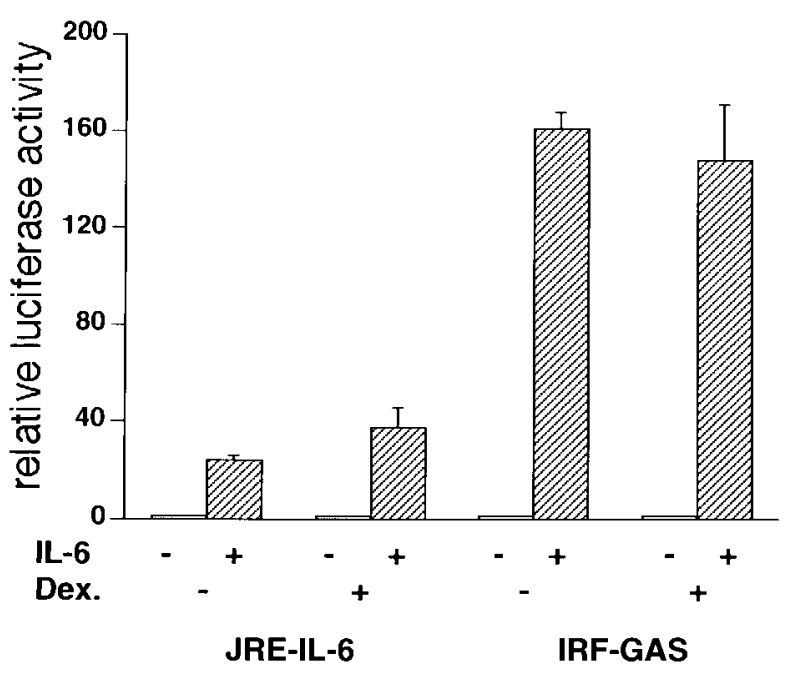

Figure 6 Effect of Dex on the IL-6-induced activation of the JRE-IL-6-luciferase gene or of the IRF-GAS-luciferase gene. MCF7 cells were transfected with the $3 \times$ JRE-IL-6-luciferase or the $3 \times$ IRF-GAS-luciferase gene. The transfected cells were incubated with vehicle $(-)$ or IL-6 $(100 \mathrm{ng} / \mathrm{ml})$ alone or in combination with Dex $\left(10^{-6} \mathrm{M}\right)$ for $6 \mathrm{~h}$, and luciferase activity was determined. Relative luciferase activity of unstimulated cells is shown as 1 . Data are averages of three independent experiments, with standard deviations indicated by bars.

not required for tyrosine phosphorylation of STAT3 and is specific for APRE may be the target of the glucocorticoid signaling pathway.

Glucocorticoid does not change the mobility of IL-6-induced APRE-binding proteins

It is possible that Dex treatment may synthesize a new STAT3 interactive protein and enhance the IL-6-induced APRE activation. Therefore we examined whether Dex treatment changes the mobility of IL-6-induced APREbinding proteins using EMSAs (Fig. 7). IL-6-induced APRE-binding activity was detected at 15 min stimulation but it was not detected at $6 \mathrm{~h}$ with or without Dex treatment (Fig. 7, lanes 2-5). Dex treatment did not change the mobility of IL-6-induced APRE-binding proteins but increased the binding activity by approximately 1.8-fold (Fig. 7, lanes 2 and 3). The IL-6 and Dex-induced APRE-binding complex was supershifted by anti-STAT3 antibody, whereas anti-GR antibody had no effect (Fig. 7 , lanes 6 and 7). These results suggest that Dex does not synthesize the STAT3-binding protein and GR is not involved in the IL-6 and Dex-induced APRE-binding proteins.

\section{Discussion}

Here we have shown that: (1) Dex synergistically activated the IL-6-induced APRE-luciferase gene activation,

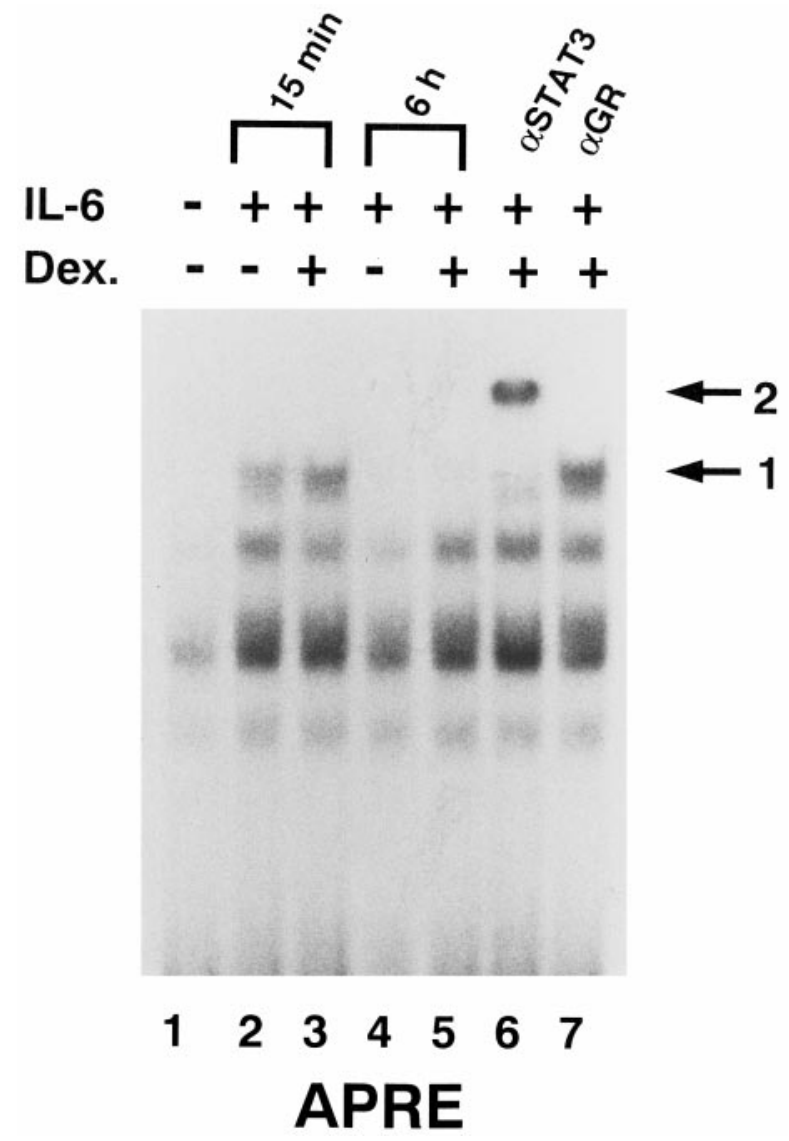

Figure 7 Effect of Dex on the IL-6-induced APRE-binding proteins. MCF7 cells were stimulated with vehicle $(-$ ) (lane 1) or IL-6 $(100 \mathrm{ng} / \mathrm{ml})$ alone (lanes 2 and 4$)$ or in combination with Dex $\left(10^{-6} \mathrm{M}\right)$ (lanes 3 and 5-7) for the indicated time, then nuclear extracts were prepared. For each reaction, $8 \mu \mathrm{g}$ nuclear extract were incubated with a ${ }^{32} \mathrm{P}$-labeled APRE probe. The reactions were followed by electrophoresis in a $4.5 \%$ polyacrylamide gel. Where indicated, anti-STAT3 antibody ( $\alpha$ STAT3, lane 6 ) or anti-GR antibody ( $\alpha \mathrm{GR}$, lane 7$)$ was added. Arrow 1 indicates the position of the IL-6-induced APRE-binding proteins. Arrow 2 indicates the position of the supershifted complex of the IL-6-induced APRE-binding proteins.

(2) the exogenous expression of DN-STAT3 completely abolished the IL-6 plus Dex-induced APRE activation, (3) the level and time course of IL-6-induced STAT3 tyrosine phosphorylation were not changed by Dex treatment, (4) the synergism was not detected in other IL-6 response elements (JRE-IL-6, IRF-GAS) which were also activated by tyrosine phosphorylated STAT3, and (5) Dex treatment did not change the mobility of the IL-6induced APRE-binding proteins in EMSAs.

For the functional characterization of the acute phase protein genes, the human hepatoma cell line HepG2 is most frequently used (Baumann et al. 1987, 1990). At first we analyzed the synergism by using HepG2 cells, but the synergistic effect was only $1 \cdot 3$-fold (data not shown). It has 
been reported that the expression level of GR in HepG2 cells is very low (Hocke et al. 1992), so we chose MCF7 cells, which have functional GR (Slater et al. 1991). As shown in Fig. 1A the synergistic effect was $2 \cdot 4$-fold in MCF7 cells.

The steroid receptor superfamily and STAT transcription factors are different sets of signaling molecules and activate gene transcription by binding to their respective responsive elements (Beato et al. 1995, Nakajima et al. 1995). Therefore these signal transduction pathways had seemed to be unrelated. But recently synergy between STAT5 and GR was reported in the activation of $\beta$-casein gene transcription (Stocklin et al. 1996, Lechner et al. 1997). STAT5 and GR form a molecular complex, which is co-precipitated with STAT5 or GR antiserum (Stocklin et al. 1996). They cooperate to activate transcription by binding to the STAT5-binding site. In our case it is also possible that STAT3 and GR form a complex. But we could not detect the complex formation in a coimmunoprecipitaion experiment using antibody against STAT3 or GR (data not shown). The facts that the synergy between IL-6 and Dex was restricted to the activation of the APRE site, and that other STAT3binding sites (JRE-IL-6 and IRF-GAS) were not synergistically activated, also supported the data that the complex formation between STAT3 and GR was not observed. EMSA analysis in this study showed that Dex treatment did not change the mobility of the IL-6-induced APRE-binding proteins. These results provide further support that a complex between STAT3 and GR is not formed.

JRE-IL-6 and IRF-GAS sites consist of two cooperative DNA motifs; one is a STAT-binding site (JRE-IL-6: TTCCTGACA, IRF-GAS: TTCCCCGAA) and the other a cAMP responsive element (CRE)-like site (JREIL-6: TGACGCGA, IRF-GAS: TGACGGCA) (Kojima et al. 1996). The presence of binding proteins to the CRE-like site besides STAT3 has been reported (Kojima et al. 1996). As the APRE site consists only of a STATbinding site (TTCTGGGAA) and does not contain a CRE-like site, the mechanism of IL-6-induced APRE activation seemed to be different from that in JRE-IL-6 and IRF-GAS. So it is possible that the difference in Dex effect on IL-6-induced IL-6 response element activation is due to the differences in the response elements among these genes.

We have shown the presence of an unknown coactivation process which was needed for leukemia inhibitory factor (LIF)-induced APRE activation. The coactivation mechanism was not required for tyrosine phosphorylation of STAT3 (Takeda et al. 1997a). As LIF uses the same JAK-STAT (STAT3) signal transduction pathway as IL-6 (Hirano et al. 1994), this coactivation process may be present in IL-6-induced APRE activation. In our study Dex treatment did not change the level of IL-6-induced STAT3 phosphorylation, therefore it is possible that Dex may enhance the activity of this coactivation process. Further examination of this mechanism will provide a new insight into the regulation of IL-6 response element activation by glucocorticoid.

\section{Acknowledgements}

We are grateful to Dr T Hirano, Osaka University Medical School, Osaka, Japan for the gift of the IL-6 and to Dr K Nakajima, Osaka University Medical School, Osaka, Japan for providing the dominant negative STAT3 expression vector.

\section{References}

Baumann H 1989 Hepatic acute phase reaction in vivo and in vitro. In Vitro Cellular and Developmental Biology 25 115-126.

Baumann H, Richards C \& Gauldie J 1987 Interaction among hepatocyte-stimulating factors, interleukin 1, and glucocorticoids for regulation of acute phase plasma proteins in human hepatoma (HepG2) cells. Journal of Immunology 139 4122-4128.

Baumann H, Jahreis GP \& Morella KK 1990 Interaction of cytokineand glucocorticoid-response elements of acute-phase plasma protein genes. Importance of glucocorticoid receptor level and cell type for regulation of the elements from rat alpha 1-acid glycoprotein and beta-fibrinogen genes. Journal of Biological Chemistry 265 22275-22281.

Beato M, Herrlich P \& Schutz G 1995 Steroid hormone receptors: many actors in search of a plot. Cell 83 851-857.

Evans RM 1988 The steroid and thyroid hormone receptor superfamily. Science 240 889-895.

Gauldie J, Richards C, Northemann W, Fey G \& Baumann H 1989 IFN beta $2 / \mathrm{BSF} 2 / \mathrm{IL}-6$ is the monocyte-derived HSF that regulates receptor-specific acute phase gene regulation in hepatocytes. Annals of the New York Academy of Sciences 557 46-58.

Hirano T, Matsuda T \& Nakajima K 1994 Signal transduction through gp130 that is shared among the receptors for the interleukin 6 related cytokine subfamily. Stem Cells 12 262-277.

Hocke GM, Barry D \& Fey GH 1992 Synergistic action of interleukin- 6 and glucocorticoids is mediated by the interleukin-6 response element of the rat alpha 2 macroglobulin gene. Molecular and Cellular Biology 12 2282-2294.

Hollenberg SM, Giguere V, Segui P \& Evans RM 1987 Colocalization of DNA-binding and transcriptional activation functions in the human glucocorticoid receptor. Cell 49 39-46.

Kojima H, Nakajima K \& Hirano T 1996 IL-6-inducible complexes on an IL-6 response element of the junB promoter contain Stat3 and $36 \mathrm{kDa}$ CRE-like site binding protein(s). Oncogene 12 547-554.

Lechner J, Welte T, Tomasi JK, Bruno P, Cairns C, Gustafsson J \& Doppler W 1997 Promoter-dependent synergy between glucocorticoid receptor and Stat5 in the activation of beta-casein gene transcription. Journal of Biological Chemistry 272 20954-20960.

Lutticken C, Wegenka UM, Yuan J, Buschmann J, Schindler C, Ziemiecki A, Harpur AG, Wilks AF, Yasukawa K, Taga T, Kishimoto T, Barbieri G, Pellegrini S, Sendtner M, Heinrich PC \& Horn F 1994 Association of transcription factor APRF and protein kinase Jak1 with the interleukin-6 signal transducer gp130. Science 263 89-92.

Nakajima K, Kusafuka T, Takeda T, Fujitani Y, Nakae K \& Hirano $\mathrm{T} 1993$ Identification of a novel interleukin-6 response element containing an Ets-binding site and a CRE-like site in the junB promoter. Molecular and Cellular Biology 13 3027-3041. 
Nakajima K, Matsuda T, Fujitani Y, Kojima H, Yamanaka Y, Nakae K, Takeda T \& Hirano T 1995 Signal transduction through IL-6 receptor: involvement of multiple protein kinases, stat factors, and a novel H7-sensitive pathway. Annals of the New York Academy of Sciences 762 55-70.

Nakajima K, Yamanaka Y, Nakae K, Kojima H, Ichiba M, Kiuchi N, Kitaoka T, Fukada T, Hibi M \& Hirano T 1996 A central role for Stat3 in IL-6-induced regulation of growth and differentiation in M1 leukemia cells. EMBO Journal 15 3651-3658.

Nishio Y, Isshiki H, Kishimoto T \& Akira S 1993 A nuclear factor for interleukin-6 expression (NF-IL6) and the glucocorticoid receptor synergistically activate transcription of the rat alpha 1-acid glycoprotein gene via direct protein-protein interaction. Molecular and Cellular Biology 13 1854-1862.

Ruff Jamison S, Chen K \& Cohen S 1993 Induction by EGF and interferon-gamma of tyrosine phosphorylated DNA binding proteins in mouse liver nuclei. Science 261 1733-1736.

Sadowski HB, Shuai K, Darnell JE Jr \& Gilman MZ 1993 A common nuclear signal transduction pathway activated by growth factor. Science 261 1739-1744.

Slater EP, Redeuilh G \& Beato M 1991 Hormonal regulation of vitellogenin genes: an estrogen-responsive element in the Xenopus A2 gene and a multihormonal regulatory region in the chicken II gene. Molecular Endocrinology 5 386-396.

Stocklin E, Wissler M, Gouilleux F \& Groner B 1996 Functional interactions between Stat5 and the glucocorticoid receptor. Nature 383 726-728.

Takeda T, Nakajima K, Kojima H \& Hirano T 1994 E1A repression of IL-6-induced gene activation by blocking the assembly of IL-6 response element binding complexes. Journal of Immunology 153 4573-4582.

Takeda T, Kurachi H, Yamamoto T, Homma H, Adachi K, Morishige K, Miyake A \& Murata Y 1997a Alternative signaling mechanism of leukemia inhibitory factor responsiveness in a differentiating embryonal carcinoma cell. Endocrinology 138 2689-2696.

Takeda T, Kurachi H, Yamamoto T, Homma H, Morishige K, Miyake A \& Murata Y $1997 b$ Participation of JAK, STAT and unknown proteins in human placental lactogen-induced signaling: a unique signaling pathway different from prolactin and growth hormone. Journal of Endocrinology 153 R1-R3.

Wegenka UM, Buschmann J, Lutticken C, Heinrich PC \& Horn F 1993 Acute-phase response factor, a nuclear factor binding to acute-phase response elements, is rapidly activated by interleukin- 6 at the posttranslational level. Molecular and Cellular Biology 13 276-288.

Zhang X, Blenis J, Li HC, Schindler C \& Chen Kiang S 1995 Requirement of serine phosphorylation for formation of STAT-promoter complexes. Science 267 1990-1994.

Zhong Z, Wen Z \& Darnell JE Jr 1994 Stat3: a STAT family member activated by tyrosine phosphorylation in response to epidermal growth factor and interleukin-6. Science 264 95-98.

Received 23 April 1998

Accepted 8 July 1998 